\title{
Multi Objective Geometric Programming with Interval Coefficients: A Parametric Approach
}

\author{
Zeinab Mousavi ${ }^{1}$ and Mansour Saraj ${ }^{2}$ \\ ${ }^{1}$ Department of Mathematics, Faculty of Mathematical Sciences and Computer, \\ Shahid Chamran University of Ahvaz, Ahvaz, Iran; e-mail: zeinabmousavi869@gmail.com \\ ${ }^{2}$ Department of Mathematics, Faculty of Mathematical Sciences and Computer, \\ Shahid Chamran University of Ahvaz, Ahvaz, Iran; e-mail: msaraj@scu.ac.ir
}

\begin{abstract}
When we talk of optimization in industry we need to pay attention in searching for very powerful and flexible optimization techniques. One of such techniques which has attracted the interest of many researchers in the last few decades is called geometric programming that provides a powerful tool for solving nonlinear problems. As we know in the real world, many applications of geometric programming are engineering design problems. Generally, engineering design problems deal with multi-objective functions, in which their objectives are often in conflicts with each other. This paper considers a solution method when the cost, the constraint coefficients, and the right-hand sides in the multi-objective geometric programming problems are imprecise and represented as interval values. This problem is reduced with the method of weighted sum to a single objective function and further by applying interval-valued function, we solve the problem by geometric programming technique. The ability of calculating the bounds of the objective value developed in this paper might help lead to more realistic modeling efforts in engineering optimization areas. Finally a numerical example is given to illustrate the methodology of solution and efficiency of the present approach.
\end{abstract}

Received: May 18, 2019; Revised: August 7, 2019; Accepted: August 10, 2019

2010 Mathematics Subject Classification: 90C29, 90C30, 90C86.

Keywords and phrases: multi-objective programming, geometric programming, weighting method, parametric interval.

Copyright (C) 2019 Zeinab Mousavi and Mansour Saraj. This is an open access article distributed under the Creative Commons Attribution License, which permits unrestricted use, distribution, and reproduction in any medium, provided the original work is properly cited. 


\section{Introduction}

Geometric Programming (GP) is a methodology for solving algebraic nonlinear optimization problems especially found in engineering design and manufacturing. The theory of GP was introduced by Duffin et al. [2] to put a foundation stone to solve a wide range of engineering design problems. The most important property of GP is that a problem with highly nonlinear constraints can be stated easily and reduced to a problem with only linear constraints, with the help of its deal, since linear constraints are obviously effective in making a problem easier to some extent. For more detail on discussions of various algorithms and computational aspects for GP, refers to Beightler and Phillips [1].

The problem parameters of many applications of geometric programming problems are estimates of actual values (Beightler and Philips [1]). In real World, to get the exact information regarding parameters data collection cannot be possible due to human errors or some unexpected situations. Therefore, there are many cases in which these parameters may not be presented in their exact sense. The main approach is to apply interval estimates instead of single values to represent the uncertain parameter. Liu [3] developed a solution method of posynomial geometric programming with interval exponents and coefficients. A pair of two-level mathematical programs is formulated, and hence it solves the pair of problems using the geometric programming technique to obtain the objective value as an interval number. Mahapatra and Mandal [4] developed a solution procedure using GP technique by applying the interval-valued function as coefficients. Generally, an engineering design problem has multiple objective functions. In this case, it is not suitable to use any single objective programming to find an optimal compromise solution. Ojha and Ota [5] developed a method to solve multi-objective Geometric programming problem where cost coefficients of objective function as well as coefficients of constraints are multiple parameters. Saraj and Bazikar [6] solved the problem of multi-objective geometric programming problem via reference point approach. Mahapatra and Mandal [7] introduced parametric functional form and then solved the problem by geometric programming technique. Ojha and Das [8] considered a solution procedure to solve nonlinear programming problems using GP technique by splitting the cost coefficients and exponents with the help of binary numbers. Ojha and Biswal [9] developed a new method to solve geometric programming problems with multiple parameters exponents and coefficients, in which the equivalent mathematical 
programming on using a variable separable technique based on the duality theorem yields to multiple objective values. Ojha and Das [10] investigated an optimization problem where the cost coefficients are continuous functions and applied weighted method to obtain the non-inferior solutions. Das and Roy [11] developed a methodology to solve a multi-objective geometric programming by applying MOGP technique based on weighted-sum method, weighted-product and min-max method. Ojha and Biswal [12] worked out on basic concepts and principal of multiple objective optimization problems and they developed a new technique to solve geometric programming by using weighted mean to get the non-inferior solution to the problem.

In this paper, we have emphasized on multi-objective posynomial geometric programming problems and developed a solution method which will be able to calculate the bounds of objective value for the problems where the cost, the constraint coefficients and right-hand sides are interval parameters. The ability of calculating the bounds of objective value is basically developed in this paper which is the extension of [5] that may help researchers in constructing more realistic model in optimization field. Weighting method has been applied to the problems and by use of interval-valued function, we have been able to solve the problem by geometric programming technique. Very pioneer concept on interval Analysis is due to Moore et al. [13]. Wu [14] in which they proposed Psareto optimal solutions for the multi-objective problems with interval-valued functions, by considering the continuity and differentiability of an interval-valued function and the KKT optimality conditions. Mandal and Islam [15] by applying a parametric interval-valued functional form, solved the parametric geometric programming problem. Marler and Arora [16] verified the fundamental significance of the weights in terms of preferences, the Pareto optimal set and objective-function values. Liu [17] proposed the profit-maximization problem with interval coefficients and input quantity discount then by using the two-level mathematical programming achieve the upper and lower bound of the profit value. Bhurjee and Panda [18] considered a nonlinear multi-objective problem whose parameter in the objective function and constraints between lower and upper bounds. Liu [19] developed a strong tool for solving nonlinear problems where nonlinear relations stated by exponential function. $\mathrm{Xu}$ [20] provided a global optimization approach for solving signomial geometric programming problems. 


\section{Formulation of Multi-objective Geometric Programming Problem (MOGPP)}

A multi-objective geometric programming problem can be defined as:

Find $x=\left(x_{1}, x_{2}, \ldots, x_{n}\right)^{T}$ so as to

$\operatorname{Min} \hat{f}_{k o}(x)=\sum_{t=1}^{s_{k o}} c_{k o t} \prod_{j=1}^{n} x_{j}^{a_{k o t j}} \quad k=1,2, \ldots, p$

subject to

$$
\begin{aligned}
& \hat{f}_{i}(x)=\sum_{t=1}^{s_{i}} c_{i t} \prod_{j=1}^{n} x_{j}^{\gamma_{i t j}} \leq b_{i} \quad i=1,2, \ldots, m \\
& x_{j}>0, \quad j=1,2, \ldots, n,
\end{aligned}
$$

where

$c_{k o t}>0, c_{i t}>0$ for all $k, t$ and $i$.

$a_{k o t j}$ and $\gamma_{i t j}$ are real numbers for all $i, j, k, t$.

$s_{k o}=$ Number of terms present in the $k$-th objective function.

$s_{i}=$ Number of terms present in the $k$-th constraints.

All the coefficients in Model (2.1) must be precise. If any of the coefficients be represented as an interval value, therefore we expect that the objective value to be imprecise as well. Suppose $\hat{c}_{k o t}, \hat{c}_{i t}, \hat{b}_{i}$ denote the interval counterparts of $c_{k o t}, c_{i t}$ and $b_{i}$, respectively. The MOGPP with interval coefficients is of the following form:

Find $x=\left(x_{1}, x_{2}, \ldots, x_{n}\right)^{T}$ so as to

$\operatorname{Min} \hat{f}_{k o}(x)=\sum_{t=1}^{s_{k o}} \hat{c}_{k o t} \prod_{j=1}^{n} x_{j}^{a_{k o t j}} \quad k=1,2, \ldots, p$

subject to

$$
\hat{f}_{i}(x)=\sum_{t=1}^{s_{i}} \hat{c}_{i t} \prod_{j=1}^{n} x_{j}^{\gamma_{i t j}} \leq \hat{b}_{i} \quad i=1,2, \ldots, m
$$




$$
x_{j}>0, \quad j=1,2, \ldots, n,
$$

where

$$
\begin{aligned}
& \hat{c}_{k o t} \in\left[c_{k o t}^{L}, c_{k o t}^{U}\right], \quad \hat{c}_{i t} \in\left[c_{i t}^{L}, c_{i t}^{U}\right], \hat{b}_{i} \in\left[b_{i}^{L}, b_{i}^{U}\right], \\
& c_{k o t}^{L}>0, \quad c_{i t}^{L}>0 \text { and } b_{i}^{L}>0, \forall k, i, t .
\end{aligned}
$$

\section{Weighting Method Solution of MOGPP}

As we know the weighting method is one of the most popular techniques for solving MOGPPS, which can be applied to obtain the non inferior optimal solution of multiobjective functions with the convex objective space. A weighting method for MOGPP with interval coefficients can be defined as follows:

$$
(P): \operatorname{Min} \sum_{k=1}^{p} w_{k} \hat{f}_{k o}(x)=\sum_{k=1}^{p} w_{k}\left[\sum_{t=1}^{s_{k o}} \hat{c}_{k o t} \prod_{j=1}^{n} x_{j}^{a_{k o t j}}\right]
$$

subject to

$$
\begin{aligned}
& \hat{f}_{i}(x)=\sum_{t=1}^{s_{i}} \hat{c}_{i t} \prod_{j=1}^{n} x_{j}^{\gamma_{i t j}} \leq \hat{b}_{i} \quad i=1,2, \ldots, m \\
& x_{j}>0 \quad j=1,2, \ldots, n \\
& \sum_{k=1}^{p} w_{k}=1 \quad w_{k}>0 \quad k=1,2, \ldots, p .
\end{aligned}
$$

\section{Mathematical Formulation}

Definition 4.1. Let $a>0, b>0$ and consider the interval $[a, b]$. As we know mathematically any real number can be considered as a line, therefore in a similar fashion, we can represent an interval by a function. If we represent an interval in the form of $[a, b]$, then corresponding interval-valued function can be assumed as:

$$
H(q)=a^{1-q} b^{q} \text { for } q \in[0,1] .
$$


The model $(\mathrm{P})$ can be written in the form of:

$$
(P q): \operatorname{Min} \sum_{k=1}^{p} w_{k}\left[\sum_{t=1}^{s_{k o}}\left(c_{k o t}^{L}\right)^{1-q}\left(c_{k o t}^{U}\right)^{q} \prod_{j=1}^{n} x_{j}^{a_{k o t j}}\right]
$$

subject to

$$
\begin{aligned}
& \hat{f}_{i}(x)=\sum_{t=1}^{s_{i}}\left(c_{i t}^{L}\right)^{1-q}\left(c_{i t}^{U}\right)^{q} \prod_{j=1}^{n} x_{j}^{\gamma_{i t j}} \leq \hat{b}_{i}, \quad i=1,2, \ldots, m \\
& x_{j}>0 \quad j=1,2, \ldots, n \\
& \sum_{k=1}^{P} w_{k}=1, \quad w_{k}>0, \quad k=1,2, \ldots, p \\
& 0 \leq q \leq 1 .
\end{aligned}
$$

The following theorem gives the idea that in fact both the solution of the model (P) and the model $(\mathrm{Pq})$ are same when the coefficients are the interval values of the MOGPP.

Theorem 4.2. The problem $(P q)$ provides the solution of the problem $(P)$.

Proof. The given model (P) can be written as follows:

$$
\operatorname{Min} \sum_{k=1}^{p} w_{k}\left[\sum_{t=1}^{s_{k o}}\left[c_{k o t}^{L}, c_{k o t}^{U}\right] \prod_{j=1}^{n} x_{j}^{a_{k o t j}}\right]
$$

subject to

$$
\begin{aligned}
& \sum_{t=1}^{s_{i}}\left[c_{i t}^{L}, c_{i t}^{U}\right] \prod_{j=1}^{n} x_{j}^{\gamma_{i t j}} \leq\left[b_{i}^{L}, b_{i}^{U}\right] i=1,2, \ldots, m \\
& x_{j}>0 \quad j=1,2, \ldots, n \\
& \sum_{k=1}^{p} w_{k}=1, w_{k}>0, k=1,2, \ldots, p .
\end{aligned}
$$

For any $k$ and $t$, if we take $\alpha_{k t} \in\left[c_{k o t}^{L}, c_{k o t}^{U}\right], \beta_{t} \in\left[c_{i t}^{L}, c_{i t}^{U}\right]$ and $\xi_{i} \in\left[b_{i}^{L}, b_{i}^{U}\right]$ the Problem 4.1 reduces to 


$$
\begin{aligned}
& \operatorname{Min} \sum_{k=1}^{p} w_{k}\left[\sum_{t=1}^{s_{k o}} \alpha_{k t} \prod_{j=1}^{n} x_{j}^{a_{k o t j}}\right] \\
& \sum_{t=1}^{s_{i}} \beta_{t} \prod_{j=1}^{n} x_{j}^{\gamma_{i t j}} \leq \xi_{i}, \quad i=1,2, \ldots, m \\
& x_{j}>0 \quad j=1,2, \ldots, n \\
& \sum_{k=1}^{p} w_{k}=1, w_{k}>0 \quad k=1,2, \ldots, p .
\end{aligned}
$$

For any fixed $i$, let us consider the interval-valued function $h_{i}(q)=a_{i}^{1-q} b_{i}^{q}$ for $q \in[0,1]$ for an interval $\beta_{i}, \xi_{i} \in\left[a_{i}, b_{i}\right]$ similarly. For any fixed $k$, we consider $h_{k}(q)=a_{k}^{1-q} b_{k}^{q}$ for $q \in[0,1]$ for an interval $\alpha_{k} \in\left[a_{k}, b_{k}\right]$. Since $h_{i}(q)=a_{i}^{1-q} b_{i}^{q}$ for $q \in[0,1]$ and $h_{k}(q)=a_{k}^{1-q} b_{k}^{q}$ for $q \in[0,1]$ are strictly monotone and continuous functions, its inverse exists. Let $\delta$ be the inverse of $h_{i}(q)$, the $q=\frac{\log \delta+\log a_{i}}{\log b_{i}-\log a_{i}}$; therefore, similarly suppose $v$ be the inverse of $h_{k}(q)$, then $q=\frac{\log v+\log a_{k}}{\log b_{k}-\log a_{k}}$. Thus, we can calculate any particular $\alpha_{k}, \beta_{i}, \xi_{i}$ values of $q \in[0,1]$.

The optimal solution of the problem $(\mathrm{P})$ can be obtained by solving the model $(\mathrm{Pq})$, which can be written as follows:

$$
\begin{aligned}
& \operatorname{Min} \sum_{k=1}^{p} w_{k}\left[\sum_{t=1}^{s_{k o}}\left(c_{k o t}^{L}\right)^{1-q}\left(c_{k o t}^{U}\right)^{q} \prod_{j=1}^{n} x_{j}^{a_{k o t j}}\right] \\
& \sum_{t=1}^{s_{i}}\left(c_{i t}^{L}\right)^{1-q}\left(c_{i t}^{U}\right)^{q} \prod_{j=1}^{n} x_{j}^{\gamma_{i t j}} \leq\left(b_{i}^{L}\right)^{q},\left(b_{i}^{U}\right)^{1-q} i=1,2, \ldots, m \\
& x_{j}>0 \quad j=1,2, \ldots, n \\
& \sum_{k=1}^{p} w_{k}=1 w_{k}>0, k=1,2, \ldots, p \\
& 0 \leq q \leq 1 .
\end{aligned}
$$




\section{Numerical Example}

The given example represents the ability of the proposed approach in this paper for solving a MOGPP with interval value of cost, constraint coefficients and right-hand sides.

\section{Example.}

Find $x_{1}, x_{2}, x_{3}$ so as to

$\operatorname{Min} f_{1}(x)=[0.3,1.75] x_{1}^{-1} x_{2}^{-1} x_{3}^{-1}$

$\operatorname{Min} f_{2}(x)=[0.25, \quad 2] x_{1}^{-1} x_{2}^{-3} x_{3}^{-5}+x_{1}^{-1} x_{2}^{-1}$

Subject to

$$
\begin{gathered}
{[0.2,1.2] x_{1} x_{2} x_{3}^{2}+x_{2} x_{3} \leq[4,8]} \\
x_{1} x_{3} \leq 1 \\
x_{1}, x_{2}, x_{3}>0 .
\end{gathered}
$$

Based on the model $(\mathrm{P})$, the problem can be reduced to a single objective function as:

$$
\begin{aligned}
\operatorname{Min} z= & w_{1}\left([0.3,1.75] x_{1}^{-1} x_{2}^{-1} x_{3}^{-1}\right) \\
& +w_{2}\left([0.25,2] x_{1}^{-1} x_{2}^{-3} x_{3}^{-5}+x_{1}^{-1} x_{2}^{-1}\right)
\end{aligned}
$$

subject to

$$
\begin{gathered}
{[0.2,1.2] x_{1} x_{2} x_{3}^{2}+x_{2} x_{3} \leq[4,8]} \\
x_{1} x_{3} \leq 1 \\
x_{1}, x_{2}, x_{3}>0 .
\end{gathered}
$$

Here $w_{1}+w_{2}=1, w_{1}, w_{2}>0$.

According to Theorem 4.2 problem $z$ can be transformed into the following form:

$$
\begin{aligned}
\text { Min } z_{q}= & w_{1}\left(0.3^{1-q} 1.75^{q} x_{1}^{-1} x_{2}^{-1} x_{3}^{-1}\right) \\
& +w_{2}\left(0.25^{1-q} 2^{q} x_{1}^{-1} x_{2}^{-3} x_{3}^{-5}+x_{1}^{-1} x_{2}^{-1}\right)
\end{aligned}
$$


subject to

$$
\begin{gathered}
0.2^{1-q_{1}} 1.2^{q} x_{1} x_{2} x_{3}^{2}+x_{2} x_{3} \leq 4^{q} 8^{1-q} \\
x_{1} x_{3} \leq 1 \\
0 \leq q \leq 1 \\
x_{1}, x_{2}, x_{3}>0 .
\end{gathered}
$$

Here $w_{1}+w_{2}=1, w_{1}, w_{2}>0$.

In this problem the degree of difficulty is 2 and it can be solved by the corresponding dual problem:

$$
\begin{aligned}
\operatorname{Max}\left(z_{q}\right) D= & \left(\frac{0.3^{1-q_{1}} 1.75^{q} w_{1}}{w_{01}}\right)^{w_{01}}\left(\frac{0.25^{1-q_{2} q} w_{02}}{w_{02}}\right)^{w_{02}}\left(\frac{w_{2}}{w_{03}}\right)^{w_{03}} \\
& \times\left(\frac{0.2^{1-q_{1}} \cdot 2^{q}}{4^{q} 8^{1-q} w_{11}}\right)^{w_{11}}\left(\frac{1}{4^{q^{1}} 8^{1-q} w_{12}}\right)^{w_{12}}\left(w_{11}+w_{12}\right)^{\left(w_{11}+w_{12}\right)}
\end{aligned}
$$

subject to

$$
\begin{gathered}
w_{01}+w_{02}+w_{03}=1 \\
-w_{01}-w_{02}-w_{03}+w_{11}+w_{21}=0 \\
-w_{01}-3 w_{02}-w_{03}+w_{11}+w_{21}=0 \\
-w_{01}-5 w_{02}+2 w_{11}+w_{12}+w_{21}=0 \\
w_{01}, w_{02}, w_{03}, w_{11}, w_{12}, w_{21} \geq 0 \\
0 \leq q \leq 1 \\
w_{1}, w_{2}>0 .
\end{gathered}
$$

For different values of parameter $q$ and the different weights $w_{1}, w_{2}$ the dual variables and the maximum value of dual objective function, for example, for $w_{1}=w_{2}=0.5$ is given in the following table. 
Table 1. $w_{1}=w_{2}=0.5$ and $0 \leq q \leq 1$ for dual solution.

\begin{tabular}{cccccccc}
\hline$q$ & $w_{01}$ & $w_{02}$ & $w_{03}$ & $w_{11}$ & $w_{12}$ & $w_{21}$ & $z_{D}$ \\
\hline 0.0 & 0.3278501 & 0.5573833 & 0.1147666 & 0.3524610 & 1.762306 & 0.6475390 & 0.00720723 \\
0.1 & 0.3333503 & 0.5555499 & 0.1110998 & 0.4075645 & 1.703535 & 0.5294355 & 0.106287 \\
0.2 & 0.3383289 & 0.5538904 & 0.1077808 & 0.4690070 & 1.638774 & 0.5309930 & 0.015844 \\
0.3 & 0.3427482 & 0.5524173 & 0.1048345 & 0.5368170 & 1.568018 & 0.4631830 & 0.0239003 \\
0.4 & 0.3465724 & 0.5511425 & 0.1022850 & 0.6108104 & 1.491475 & 0.3891896 & 0.0365232 \\
0.5 & 0.3497698 & 0.5500767 & 0.1001535 & 0.6905580 & 1.409595 & 0.3094420 & 0.0565995 \\
0.6 & 0.3541842 & 0.5492287 & 0.09845733 & 0.7753703 & 1.323087 & 0.2246297 & 0.0890309 \\
0.7 & 0.3541842 & 0.5486053 & 0.09721052 & 0.8643032 & 1.232907 & 0.1356968 & 0.142264 \\
0.8 & 0.3553652 & 0.5482116 & 0.09642317 & 0.951906 & 1.140233 & 0.0438094 & 0.231063 \\
0.9 & 0.3597738 & 0.5467421 & 0.09348416 & 1.0 & 1.093484 & 0.0 & 0.380728 \\
1.0 & 0.3573434 & 0.5442189 & 0.08843771 & 1.0 & 1.088438 & 0.0 & 0.628756 \\
\hline
\end{tabular}

Table 2. $w_{1}=w_{2}=0.5$ and $0 \leq q \leq 1$ for primal solution.

\begin{tabular}{ccccc}
\hline$q$ & $x_{1}$ & $x_{2}$ & $x_{3}$ & $z$ \\
\hline 0.0 & 9.515512 & 63.43647 & 0.1050915 & 0.00720724 \\
0.1 & 8.384132 & 50.49955 & 0.1192729 & 0.106287 \\
0.2 & 7.352717 & 39.81305 & 0.1360042 & 0.015844 \\
0.3 & 6.420475 & 31.08001 & 0.1557517 & 0.0239003 \\
0.4 & 5.578173 & 23.99354 & 0.1792702 & 0.0365232 \\
0.5 & 4.819880 & 18.30015 & 0.2074740 & 0.0565995 \\
0.6 & 4.140047 & 13.77734 & 0.2415432 & 0.0890309 \\
0.7 & 3.533888 & 10.23081 & 0.2829744 & 0.142264 \\
0.8 & 2.996667 & 7.488917 & 0.3337040 & 0.231063 \\
0.9 & 2.391478 & 5.874228 & 0.3812018 & 0.380728 \\
1.0 & 1.817239 & 4.948107 & 0.4213112 & 0.628756 \\
\hline
\end{tabular}

Consequently, in Table 2 by considering the primal-dual relationship, the corresponding primal solution for different values of parameter $q$ and the weights $w_{1}=w_{2}=0.5$ is obtained.

While solving for different weights and different values of parameter $q$ we find out the upper bound of $z_{D}$ for dual solution which is obtained for $w_{1}=w_{2}=0.5$ and $q=1$ as $z_{D}^{u}=0.628756$ and the lower bound of $z_{D}$ is obtained for $w_{1}=w_{2}=0.5$ and 
$q=0$ as $z_{D}^{1}=0.00720723$, similarly for primal solution the upper and the lower bounds are obtained for the same values of weights $w_{1}=w_{2}=0.5$ and parameter $(q=0, q=1)$ as $z^{1}=0.00720724, \quad z^{u}=0.628756$. It is to be noted that all the calculations for different values of $q$ and $w$ is done by using Lingo software.

\section{Conclusion}

The parameters of mathematical models for many real world problems are usually stated imprecisely and this leads to the formulation of mathematical programming models with interval values. In this paper, by applying weighted method, a multiobjective geometric programming is converted to a single objective geometric programming problem in which parameters are interval valued numbers. The idea in the present article is to find the upper and the lower bounds of the objective value. The solution procedure is of GP technique by using interval-valued function. This technique will take minimal time and the problems with this procedure may help researchers for wider application in the field of engineering problems.

\section{References}

[1] C. S. Beightler and D. T. Phillips, Applied Geometric Programming, New York: John Wiley and Sons, 1976.

[2] R. J. Duffin, E. L. Peterson and C. M. Zener, Geometric Programming Theory and Application, New York: John Wiley and Sons, 1967.

[3] S. T. Liu, Posynomial geometric programming with interval exponents and coefficients, European Journal of Operation Research 186 (2008), 17-27. https://doi.org/10.1016/j.ejor.2007.01.031

[4] G. S. Mahapatra and T. K. Mandal, Posynomial parametric geometric programming with interval valued coefficient, J. Optim. Theory 154 (2012), 120-132. https://doi.org/10.1007/s10957-012-9996-6

[5] A. K. Ojha and R. R. Ota, Multi-objective geometric programming problems with cost co-efficients as multiple parameters, AMO-Advanced Modelling and Optimization 15 (2013), 815-838.

[6] F. Bazikar and M. Saraj, Solving linear multi-objective geometric programming problems via references point approach, Sains Malaysiana 43(8) (2014), 1271-1274. 
[7] G. S. Mahapatra and T. K. Mandal, Posynomial parametric geometric programming with interval valued coefficient, Journal of Optimization Theory and Applications 154(1) (2012), 120-132. https://doi.org/10.1007/s10957-012-9996-6

[8] A. K. Ojha and A. K. Das, Geometric programming problem with co-efficients and exponents associated with binary numbers, International Journal of Computer Science 7(1) (2010), 49-55.

[9] A. K. Ojha and K. K. Biswal, Posynomial geometric programming problems with multiple parameters, Journal of Computing 2(1) (2010), 84-90.

[10] A. K. Ojha and A. K. Das, Multi-objective geometric programming problem being cost coefficients as continuous function with weighted mean method, Journal of Computing 2(2) (2010), 67-73.

[11] P. T. Das and K. Roy, Multi-objective geometric programming and its application in Gravel Box problem, Journal of Global Research in Computer Science 5(7) (2014), 6-11.

[12] A. K. Ojha and K. K. Biswal, Multi-objective geometric programming problem with weighted mean method, International Journal of Computer Science and Information Security 7(2) (2010), 82-86.

[13] R. E. Moore, R. Baker Kearfott and M. J. Cloud, Introduction to Interval Analysis, Philadelphia: Society for Industrial and Applied Mathematics, 2009. https://doi.org/10.1137/1.9780898717716

[14] H. C. Wu, The Karush-Kuhn-Tucker optimality conditions in multiobjective programming problems with interval-valued objective functions, European Journal of Operational Research 196(1) (2009), 49-60. https://doi.org/10.1016/j.ejor.2008.03.012

[15] W. A. Mandal and S. Islam, Fuzzy unconstrained parametric geometric programming problem and its application, Journal of Fuzzy Set Valued Analysis 2016(2) (2016), 125139. https://doi.org/10.5899/2016/jfsva-00301

[16] R. T. Marler and J. S. Arora, The weighted sum method for multi-objective optimization: new insights, Structural and Multidisciplinary Optimization 41 (2010), 853-862. https://doi.org/10.1007/s00158-009-0460-7

[17] S. T. Liu, Using geometric programming to profit maximization with interval coefficients and quantity discount, Applied Mathematics and Computation 209(2) (2009), 259-265. https://doi.org/10.1016/j.amc.2008.12.035

[18] A. K. Bhurjee and G. Panda, Multi-objective optimization problem with bounded parameters, RAIRO-Oper. Res. 48(4) (2014), 545-558.

https://doi.org/10.1051/ro/2014023 
[19] S. T. Liu, Posynomial geometric programming with parametric uncertainty, European Journal of Operational Research 168(2) (2006), 345-353.

https://doi.org/10.1016/j.ejor.2004.04.046

[20] G. Xu, Global optimization of signomial geometric programming problems, European Journal of Operational Research 233(3) (2014), 500-510.

https://doi.org/10.1016/j.ejor.2013.10.016 